九州大学学術情報リポジトリ

Kyushu University Institutional Repository

\title{
Non-contact and Non-invasive Stress Evaluation Using Microwave Reflectometry
}

Nagae, Daisuke

Department of Applied Science for Electronics and Materials : Graduate Student

Mase, Atsushi

Art, Science and Technology Center for Cooperative Research, Kyushu University

https://doi.org/10.15017/18538

出版情報: 九州大学大学院総合理工学報告. 31 (4), pp. 8-12, 2010-03. 九州大学大学院総合理工学府 バージョン：

権利関係 : 


\title{
Non-contact and Non-invasive Stress Evaluation Using Microwave Reflectometry
}

\author{
Daisuke NAGAE ${ }^{*} 1, \dagger$ and Atsushi MASE ${ }^{*}$ \\ ${ }^{\dagger}$ E-mail of corresponding author:d-nagae8@asem.kyushu-u.ac.jp
}

(Received February 5, 2009)

\begin{abstract}
In this paper, we present a novel signal processing technique for stress evaluation using a microwave reflectometric cardiopulmonary sensing. This technique enables the heart rate variability (HRV) to be reconfigured from measurements of body-surface dynamic motion, which is subsequently used for the stress evaluation. In particular, a novel element is due mainly to a reconfiguration of the HRV from the time variation of the heartbeat frequency which is evaluated by a repetition of the maximum entropy method (MEM). An experimental setup of a microwave reflectometer is presented and several sets of real data are analyzed using a new signal processing technique, which are subsequently used for the stress evaluation. Finally, a utility of the new signal processing technique is discussed.
\end{abstract}

Key words: Cardiopulmonary sensing, ECG, Heart rate variability, Microwave reflectometry, StresS evaluation

\section{Introduction}

In recent years, the stress evaluation using the heart rate variability (HRV) has been widely recognized as advanced application to prevention of stress syndrome, detection of sleep prediction in driving a car, and so on. The HRV is generally obtained from peak intervals (R-R intervals) in an electrocardiogram (ECG). However, the evaluation method of the stress by using the ECG seems to be unsuitable as a long-time monitor since several electrodes have to be directly attached to a human body to acquire the ECG data. We report here on a new evaluation method of the stress using a microwave reflectometer which has the features of non-contact and non-invasive method.

There is a problem that must be solved to perform the evaluation of the stress by using a microwave reflectometer, since the information of vital signal is obtained from phase fluctuation of reflected waves. It is caused by the movement of a human body surface (reflection layer) consisting of the respiration, the heartbeat, and the random movement of the body. To extract the heartbeat signal from the measurement signal, for example, the respiration signal is removed by a high-pass filter

\footnotetext{
*1 Department of Applied Science for Electronics and Materials, Graduate Student

*2 Art, Science and Technology Center for Cooperative Research
}

(HPF) with cutoff frequency of, such as, $0.7 \mathrm{~Hz}$, although the random movement of the human body still can not be removed. The heartbeat signal is very small and has low signal-to-noise $(\mathrm{S} / \mathrm{N})$ ratio since the cardiac movement appears on the body surface through the bone and the connective tissue..$^{1-3)}$ For these reasons, the peak positions in the heartbeat signals obtained by a microwave reflectometer are not as clear as those observed in the ECG signals. Therefore, it is not easy to reconfigure the HRV correctly and automatically from the microwave reflectometer signal. In order to solve this problem, we have proposed the cross-correlation technique. This is a method reconfiguring the HRV from the cross-correlation function between a measurement signal and a template signal which is configured by averaging periodic waveform in a measurement signal. ${ }^{2)}$ However, it is difficult to reconfigure the HRV in real time by the cross-correlation technique, and which defies the detection of stress state timely. The purpose of this paper is to introduce a novel signal processing technique to reconfigure the HRV in real time.

\section{Stress Evaluation Based on ECG}

Generally, the evaluation of the stress is performed by analyzing the frequency spectrum of the HRV. ${ }^{2)}$, 4 ) The HRV is reconfigured 
by interpreting $R-R$ intervals in an ECG. The heartbeat interval is fluctuating all the time by reflecting autonomous nerve activity composed of sympathetic nerve activity and parasympathetic nerve activity. In the frequency spectrum of the HRV, the parasympathetic nerve activity appears as the power spectrum in the high frequency (HF) component with frequency

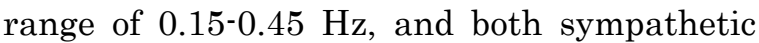
nerve activity and parasympathetic nerve activity appear in the low frequency (LF) component with frequency range of $0.03-0.15 \mathrm{~Hz}$. The peak ratio or area ratio of the power spectrum in the LF component to the HF component $(\mathrm{LF} / \mathrm{HF})$ is used to evaluate the stress, since the sympathetic nerve activity increases in a stressful state and, on the other hand, the parasympathetic nerve activity increases in a relaxed state. The value of the $\mathrm{LF} / \mathrm{HF}$ in a stressful state is larger than that in a relaxed state. For the evaluation of stress in a stationary state, the fast Fourier transform (FFT) or the maximum entropy method (MEM) has been used as a frequency analysis. In this case, the HRV data for $180 \mathrm{sec}$ is often used for the reliable estimation of stress. On the other hand, in a non-stationary state, the wavelet transform can be applied to the HRV data for the evaluation of stress.

In addition, the HRV has to be interpolated at regular intervals in derivation of the frequency spectrum. The most often-used interpolation interval is about $0.25 \mathrm{sec}$.

\section{Signal Processing}

The time variation of the heartbeat intervals can be regarded as the short-term time variation of the heartbeat frequency. In this section, the time variation of the heartbeat frequency is evaluated by applying the MEM repeatedly at the short term. The time window is shifted step by step along the temporal axis. Then, the HRV can be reconfigured since the value of the heartbeat interval is calculated by the inverse of the heartbeat frequency. The MEM is an effective method for estimating the frequency spectrum at a shot data window, and has much higher frequency resolution than the FFT.

In the MEM of analysis, the autoregressive model of observational data is estimated first, then the spectrum estimation is performed on the basis of it. The autoregressive model is given by

$$
x_{k}=-\sum_{i=1}^{m} a_{m i} x_{k-i}+n_{k}
$$

where $x_{k}$ is the observed time-series data interval, $n_{k}$ is the stationary white noise which is independent from $x_{t}(1<k), m$ is the order of the autoregressive model, and $a_{m i}$ is the autoregressive coefficient at the model order $m$. Now, we define the autocorrelation function of the time-series data $x_{k}$ as

$$
R_{i}=R(i \Delta t) \equiv E\left\{x_{k} x_{k-i}\right\}
$$

where $E\{\}$ is the expectation value. The autocorrelation function at lag 0 is shown as

$$
R_{0}=E\left\{x_{k}^{2}\right\}=-\sum_{i=1}^{m} a_{m i} R_{i}+E\left\{n_{k}^{2}\right\}
$$

Since $n_{k}$ and $x_{i}(i<k)$ are independent each other, the autocorrelation function at each lag is organized by

$$
\left[\begin{array}{cccc}
R_{0} & R_{1} & \cdots & R_{m} \\
R_{1} & R_{0} & & R_{m-1} \\
\vdots & & \ddots & \vdots \\
R_{m} & \cdots & \cdots & R_{0}
\end{array}\right]\left[\begin{array}{c}
1 \\
a_{m 1} \\
\vdots \\
a_{m m}
\end{array}\right]=\left[\begin{array}{c}
p_{m} \\
0 \\
\vdots \\
0
\end{array}\right]
$$

where $P_{m}$ is the variance of the stationary white noise. By applying the formula of Wiener-Khintchine to Eq.(1), the relationship between the autoregressive model $\left\{a_{k}\right\}$ and the power spectrum $S(\omega)$ is shown as

$$
S(\omega)=\frac{P_{m} \Delta t}{\left|1+\sum_{i=1}^{m} a_{m i} e^{-j \omega \Delta \Delta t}\right|^{2}}
$$

In the MEM, the model order is generally unknown. If the model order is selected lower, the estimated spectrum is smoothed and the spectrum peak which should be found in a normal situation does not appear sometimes. Conversely, if the model order is selected higher, a lot of spurious peaks appear finely in the estimated spectrum. The optimum selection of the model order is of importance in the MEM. Thus, we selected the optimum model order as 870 from the empirically-based experiences.

The heartbeat frequency is estimated by finding the largest peak value in the frequency range of $0.7-1.55 \mathrm{~Hz}(42-98 \mathrm{bmp})$ after calculating the power spectrum of some data windows by MEM. Here, the frequency $0.7-1.55 \mathrm{~Hz}$ covers sufficiently the range of heartbeat frequency for a seated human subject at rest. It is said that the heart rate for a seated human subject is typically in the range of $45-90 \mathrm{bmp}$, i.e., $0.75-1.5 \mathrm{~Hz}^{3)}$ 
If the analyzing data window is selected long, time response of the heartbeat intervals becomes sluggish in the HRV while the reliability of the estimation enlarges. This could exert a bad influence upon the estimation of LF/HF. In this paper, $2.5 \mathrm{sec}$ is selected as the optimum length of the data window for the MEM analysis from the empirically-based experiences.

In addition, $0.25 \mathrm{sec}$ is selected as the shift quantity of the data window. It is equal to the interpolated value of the HRV reconfigured by the ECG. The MEM is the stationary spectrum analysis technique where the consistency of the signal in the data window is assumed. Therefore, since a correspondence relationship between the data windows is ignored, some spectrum estimation errors consist by the selected data window and appear as the fluctuation by $0.25 \mathrm{sec}$ ( $4 \mathrm{~Hz}$ in frequency) in the HRV. However, it is no problem for the evaluation of the stress because the spectrum estimation error between data windows is very small and its fluctuation does not flop over the frequency domain of the LF and the HF components.

For these reasons, the time variation of the heartbeat frequency is estimated by applying the MEM repeatedly at the $2.5 \mathrm{sec}$ data window which is shifted by $0.25 \mathrm{sec}$ along the temporal axis.

\section{Experimental Results}

\subsection{Experimental setup}

Figure 1 shows a schematic diagram of the measurement system. We select a thigh as an irradiation point of microwave, since its skin movement is large due to the existence of thick artery, while it should be small during the respiration. It is not a problem whether the measurement point is right thigh or left one. We install a 16-elements patch antenna into a seat of a chair under the left thigh of a seated human subject. The back of the chair can also be selected as the antenna position since it is close to the heart. The analog output from the microwave reflectometer is fed to a HPF in order to remove the respiratory components. The output signal is then converted into digital signal using the sampling frequency $1000 \mathrm{~Hz}$ by a high-resolution (16 bits) memory scope. The digitized signals are fed into a computer, and the heartbeat signals are calculated by the detection of phase differences..$^{1-3)}$ Afterward, the evaluation of the stress is performed by applying the proposed algorithms to the calculated heartbeat signals.

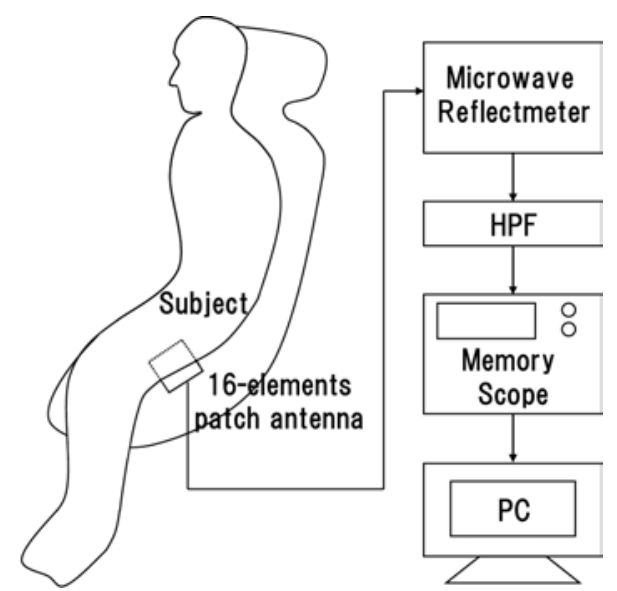

Fig.1 Experimental setup.

\subsection{Stress evaluation: static environment}

In the first experiment, the evaluation methods of the stress are applied to a person in both relaxed state and stressful state. The flash mental calculation is used to put stress on a person, which is a method that the person adds up figures displayed on a monitor continually in his head. The heartbeat measurement by a microwave reflectometer is applied to the person who is in the relaxed state or the stressful state for $180 \mathrm{sec}$. Two types of proposed methods (the cross-correlation technique and the MEM technique) are applied to reconfiguration of the HRV. Then, the LF/HF value is calculated by applying FFT to the HRV for time interval of $180 \mathrm{sec}$. For reference, the ECG data are measured at the same time in each state and the LF/HF is calculated by the conventional evaluation method of the stress.

Figure 2 shows the time evolutions of the microwave reflectometer signal and the ECG signal of the person in the relaxed state. Note that the time interval of the periodic signal is in good agreement between two signals. This means that the heart beat can be obtained correctly from the microwave reflectometry. Figure 3 shows the subsets of the HRVs calculated by the original microwave reflectometer signal and by two proposed methods together with the one obtained from the ECG. The HRVs calculated by two proposed methods agree well with that by the ECG, comparing to that by the unprocessed reflectometer signal. Additionally, the HRV calculated by the MEM contains periodical noise component compared to that calculated by the cross-correlation technique. This might be caused by some spectrum estimation errors between data windows which appear as the fluctuation by $0.25 \mathrm{sec}$ ( $4 \mathrm{~Hz}$ in frequency) in the HRV. How- 
ever, the estimated error of the HRV due to the spectrum estimation error is very small, i.e., about several percent of the HRV values. Thus the fluctuation of estimation errors is not a problem for the evaluation of the stress as described in Sec. 3. Therefore, it is understood that the HRV is reconfigured correctly by using the proposed method.

The frequencies of the HRVs reconfigured by two proposed methods and the ECG in the relaxed state are analyzed, respectively. Generally, it is said that the individual variability of the $\mathrm{LF} / \mathrm{HF}$ is about $\pm 30 \% .^{5}$ ) If the spectral shapes of the HRVs are similar and the LF/HFs can be estimated by two proposed methods in the range of $\pm 30 \%$ as compared to the ECG, it could be argued that the estimation of stress is successful.

The results are shown in Fig. 4. In the same way, the result in the stressful state is shown in Fig. 5. Here, In Figs. 4 and 5, the maximum values of the spectrum peaks are made fit with each other and normalized into 1 in order to compare three spectra. The frequency spectra of the HRVs reconfigured by applying two proposed methods to the microwave measurement are nearly conformable to that reconfigured by the ECG, respectively. The $\mathrm{LF} / \mathrm{HF}$ values by two proposed methods lie in the range of $\pm 30 \%$ compared to that obtained by the ECG in each state. Here, the LF/HF value is obtained from area values of the spectrum. In addition, it is understood that the flash mental calculation put stress on the person since the $\mathrm{LF} / \mathrm{HF}$ value in the stressful state is about twice larger than that in the relaxed state. Those results show that the evaluation of the stress can be performed by applying two proposed methods to the microwave reflectometer signal similar to the ECG.

\subsection{Stress evaluation: non-static environ- ment}

In the second experiment, the evaluation methods of the stress are applied to a person in non-static environment. It is not always true that the stress evaluation is applied to static environment in any time. In order to search the applicable scope of the system, the system is installed in a car. The stress evaluation is performed during the engine vibration is added. The heartbeat measurement by using the microwave reflectometer is applied to the person who is sitting on a driver's seat.

The two proposed methods are applied to the reconfiguration of the HRV. Then, the LF/HF value is calculated by applying the FFT to the

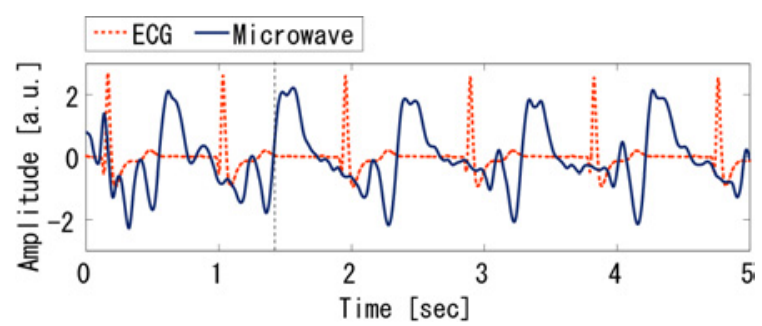

Fig.2 Comparison between the microwave reflectometer signal and the ECG signal.

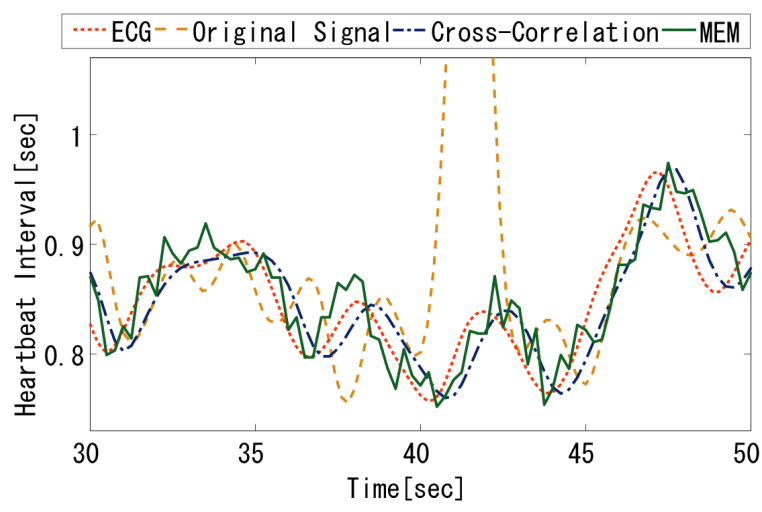

Fig.3 HRV reconfigured by each signal.

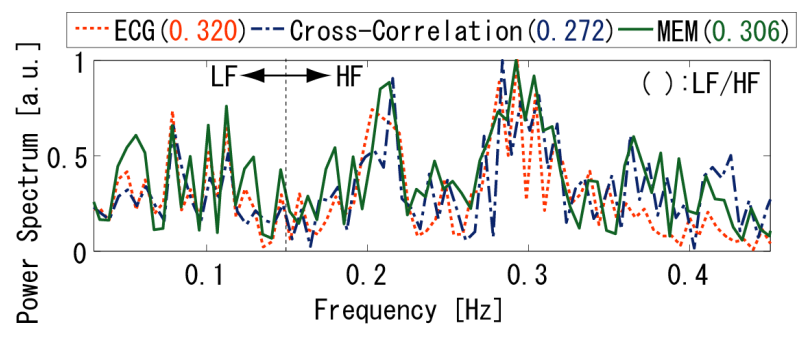

Fig.4 FFT spectrum of the HRV in relaxed state.

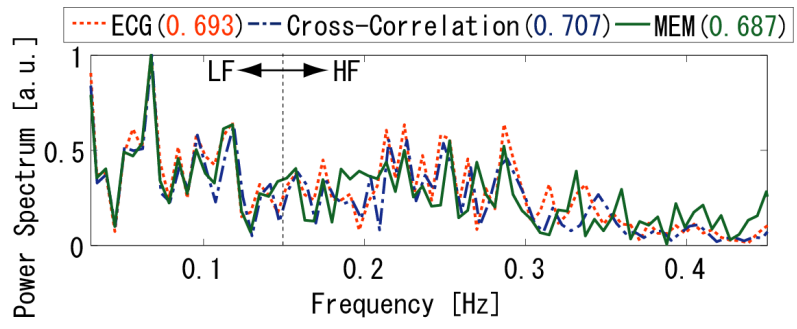

Fig.5 FFT spectrum of the HRV in the stressful state.

HRV for 180 sec. For reference, the ECG data are measured at the same time in each state and the $\mathrm{LF} / \mathrm{HF}$ value is calculated by the method.

In the non-static environment, the microwave reflectometer signal includes various noise (spurious) components due to random movements of the body surface.

They mask the heartbeat signal, and make the detection of the heartbeat interval difficult. Figure 6 shows the example of the measurement with such noise components. We inves- 
tigate whether the evaluation of stress is possible or not even in the non-static environment by two proposed methods.

Figure 7 shows the frequency spectra of the HRV reconfigured by two proposed methods together with those obtained from the ECG signals. In this case, the calculation of the cross-correlation was repeated three times to make the peak intervals of the waveform clear [2]. The spectral shapes of the HRV using the third cross-correlation function and MEM technique is nearly equal to that by the ECG and their $\mathrm{LF} / \mathrm{HF}$ s lie in the range of $\pm 30 \%$ compared to that obtained by the ECG.

Those results show that the evaluation of the stress can be performed by applying two proposed methods to microwave reflectometer signals similar to the ECGs even in non-static environment.

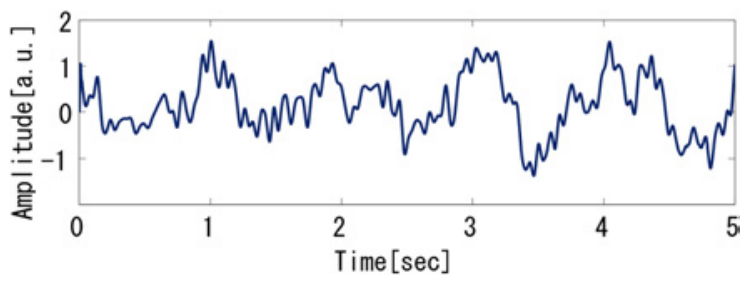

Fig.6 Time evolution of the microwave reflectometer signal.

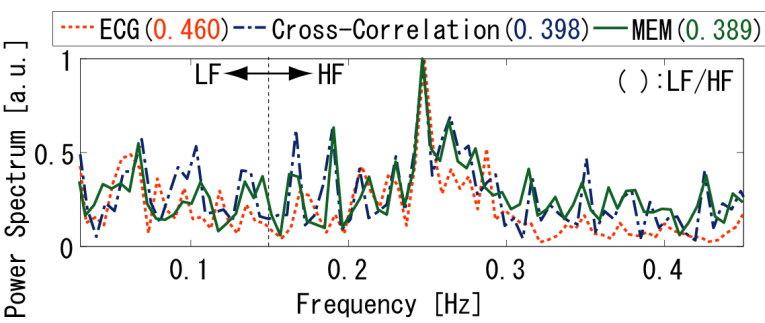

Fig.7 FFT spectra of the HRV reconfigured by the MEM, the cross-correlation technique and the ECG signal.

\subsection{Comparative discussion}

We discuss here on the utility of the new proposed method comparing with the previous one. In the previous proposed method, the microwave original signal for the first several tens sec is required at least to improve the accuracy of the template signal, and which add the time lag for several tens sec to the time variation of stress. Additionally, the repetitive operations of the cross-correlation function are required in non-static environment as shown above. These operations append large amount of calculation and complicate the processing. Thus, it could be difficult to get real-time measurement of stress by the cross-correlation technique.

On the other hand, the HRV can be reconfigured timely by $0.25 \mathrm{sec}$ using the MEM technique. The MEM technique also enables the evaluation in only one calculation even in any environment.

If the real-time evaluation of stress is desired, the MEM technique could work better than the cross-correlation technique. In the future works, the detection of stress syndrome or sleep prediction will be performed during driving a car.

\section{Conclusions}

In conclusion, we have proposed a new signal processing technique for microwave reflectometer sensing that enables the estimation of mental stress from measurement of body dynamic motion. The algorithm based on the MEM is studied to reconfigure the HRV correctly and automatically from low-S/N microwave reflectometer signals. This technique enables the stress evaluation by microwave reflectometers in any environment, and indicates a possibility of the real-time evaluation of stress toward detection of stress syndrome or sleep prediction in driving a car.

\section{Acknowledgments}

This work is partly supported by the Grant-in-Aid for Scientific Research, the Ministry of Education, Science, Sports and Culture (No. 20360186), and by Grant for Practical Application of University R\&D Results under the Matching Fund Method, NEDO.

\section{References}

1) N. Tateishi, A. Mase, L. Bruskin, Y. Kogi, N. Ito, T. Shirakata, and S. Yoshida, "Microwave Measurement of Heart Beat and Analysis Using Wavelet Transform", Proc. Asia Pacific Microwave Conference, 2151-2153 (2007).

2) D. Nagae and A. Mase, "Measurement of Vital Signal by Microwave Reflectometry and Application to Stress Evaluation", to appear Proc. Asia Pacific Microwave Conference, (2009).

3) Dennis R. Morgan, Michael G. Zierdt, "Novel signal processing techniques for Doppler radar cardiopulmonary sensing”, Signal Processing, 89, 45-66 (2009).

4) Y. Yoshida, K. Yokoyama, N. Ishii, "Real-time Continuous Assessment Method for Mental and Physiological Condition using Heart Rate Variability", IEEJ Transactions on Electronics, Information and Systems, 126-12, 1441-1446 (2006).

5) A. Kagaya, Y. Nakamura, "Regulation of Blood Flow During Exercise -Present and Future-", NAP Limited, Japan (2001) pp. 93-106. 